\title{
Bacteriological profile and antibiotic sensitivity pattern in patients with urinary tract infection
}

\author{
Subedi $\mathrm{N}^{1}$, Pudasaini $\mathrm{S}^{2}$ \\ ${ }^{I}$ Department of Urology (Surgery), Nepal Medical College Teaching Hospital,Nepal \\ ${ }^{2}$ Department of Pathology, Nepal Medical College Teaching Hospital,Nepal
}

\section{Keywords: \\ Culture; \\ Escherichia coli; \\ Infection; \\ Nephrolithiasis; \\ Sensitivity; \\ Urinary tract}

\begin{abstract}
Background: Urinary tract infection is one of the common bacterial infections seeking treatment in clinical practice. A variety of organisms are associated with UTI and the most common organisms are Escherichia coli and other coliforms. Bacteriological investigations of UTI are not complete without antibiotic sensitivity test of the isolate. The aim of this study is to determine the bacteriological profile and antibiotic sensitivity patterns and their disease association.
\end{abstract}

Materials and Methods: This study was conducted in Shankarapur Hospital over a period of one year. All cases of suspected UTI sent for urine culture and sensitivity test were evaluated in this study. Disease associated with UTI, bacteriological profile and antibiotic sensitivity patterns were evaluated.

Results: A total of 974 cases were sent for urine culture and sensitivity test. The total culture positive cases were $165(17.4 \%)$. The most common age group for culture positive test was 21 - 30 years $(33.3 \%)$ followed by $31-40$ years (25.5\%). Female patients formed the majority of culture positive cases $(84.8 \%)$ and E Coli $(86.1 \%)$ was the most common organism isolated. Piperacillin- tazobactum and ceftriaxone were the most common antibiotic sensitive to the organisms. Simple uncomplicated UTI and PID were the most common indication for subjecting the patients to urine culture.

Conclusion: UTI is most commonly seen in female of reproductive age group and the most common causative organism is E coli. Culture result and antibiogram helps the clinician for specific treatment of UTI.

\section{INTRODUCTION}

Urinary tract infection (UTI) is one of the commonest infection occurring in all age groups worldwide. ${ }^{1-3}$ It is more common in females as compared to males, especially females of reproductive age group. ${ }^{4}$ It can be either community acquired or hospital acquired. The common aetiological agents are Escherichia coli (E. Coli), Pseudomonas, Proteus and Klebsiella. ${ }^{2,4,5}$

\section{Correspondence:}

Dr. Neeraj Subedi, MBBS, MS

Assistant Professor, Department of Urology (Surgery),

Nepal Medical College Teaching Hospital, Jorpati, Kathmandu, Nepal

E-mail:nsubedi76@gmail.com
Bacteriological investigations of UTI are not complete without an antibiotic sensitivity test of the isolate. Micro organism causing UTI vary in their susceptibility to antimicrobials from place to place and time to time. ${ }^{6}$

UTI can involve infection from urethra to the kidneys. Symptoms in case of cystitis include painful and frequent urination where as condition like high fever and flank pain are seen in pyelonephritis. ${ }^{7}$ Due to the empirical use of antibiotics in infectious diseases and the lack of standardisation in antimicrobial susceptibility test, resistance 
to commonly used antimicrobial agent is increasing year by year. Knowing the common isolated uropathogens and their antimicrobial susceptibility is beneficial in planning treatment protocols. ${ }^{8}$

This study was conducted to determine the bacteriological profile and antibiotic sensitivity patterns in UTI and the disease associated with it.

\section{MATERIALS AND METHODS}

This was a cross-sectional observational study conducted in Shankarapur Hospital over a period of one year (January 2015 to December 2015). Prior to the study, permission was obtained from the hospital board. Patients presenting with symptoms of UTI such as fever, dysuria, increased frequency of urination, loin pain were subjected to urine culture. Parameters like age, sex and associated disease of the patients were taken into account. Pathogens isolated and the antibiotic sensitivity was also taken into account. Required clinical history and examination findings were noted. Patients were asked to follow up with urine culture sensitivity report. Antibiotics were started according to the sensitivity report and asked to follow up after one week. All the data were manually collected and analyzed using Excel wherever necessary.

\section{RESULTS}

A total of 947 patients clinically suspected of having UTI were included in this study and sent for culture sensitivity test. Out of the total samples sent for culture sensitivity test, the culture positive cases were $165(17.4 \%)$. Out of the positive samples, female patients were $140(84.8 \%)$ and male patents were $25(15.2 \%)$

Age wise distribution for urine culture is shown in table 1. Most common age group was 21-30 years (37.2\%) followed by age group $31-40$ years $(23.7 \%)$.

Table 2 shows age wise distribution for culture positive cases. Most number of positive urine cultures was seen in age group $21-30$ years $(33.3 \%)$ and most of the patients were female.

The most common organism isolated was E Coli and second most common was K Pneumoniae. (Table 3) Piperacillintazobactum and Ceftriaxone showed the highest number of sensitivity for the isolated organism. (Table 4)

Maximum number of patients came with symptoms of simple uncomplicated UTI. Other common causes were pregnancy, PID and BPH which is shown in Table 5.

\section{DISCUSSION}

UTI is a common problem faced by clinicians in every part

\begin{tabular}{lcccc}
\multicolumn{6}{l}{ Table 1: Age wise distribution for urine culture } \\
\hline Age (years) & Male & Female & Total (numbers) & $\begin{array}{c}\text { Percentage } \\
(\%)\end{array}$ \\
\hline$<10$ & 37 & 34 & 71 & 7.5 \\
$11-20$ & 24 & 67 & 91 & 9.6 \\
$21-30$ & 27 & 325 & 352 & 37.2 \\
$31-40$ & 28 & 196 & 224 & 23.6 \\
$41-50$ & 12 & 71 & 83 & 8.8 \\
\hline $51-60$ & 10 & 48 & 58 & 6.1 \\
\hline $61-70$ & 6 & 26 & 32 & 3.4 \\
$>70$ & 10 & 26 & 36 & 3.8 \\
\hline Total & $\mathbf{1 5 4}$ & $\mathbf{7 9 3}$ & $\mathbf{9 4 7}$ & $\mathbf{1 0 0}$ \\
\hline
\end{tabular}

Table 2: Age wise distribution for culture positive cases

\begin{tabular}{lcccc}
\hline Age (years) & Male & Female & Total (numbers) & $\begin{array}{c}\text { Percentage } \\
(\%)\end{array}$ \\
\hline$<10$ & 4 & 7 & 11 & 6.7 \\
\hline $11-20$ & 2 & 9 & 11 & 6.7 \\
\hline $21-30$ & 3 & 52 & 55 & 33.3 \\
\hline $31-40$ & 7 & 35 & 42 & 25.4 \\
$41-50$ & 4 & 12 & 16 & 9.7 \\
\hline $51-60$ & 2 & 10 & 12 & 7.3 \\
$61-70$ & 1 & 5 & 6 & 3.6 \\
$>70$ & 2 & 10 & 12 & 7.3 \\
\hline Total & $\mathbf{2 5}$ & $\mathbf{1 4 0}$ & $\mathbf{1 6 5}$ & $\mathbf{1 0 0}$ \\
\hline
\end{tabular}

Table 3: Pathogenic microorganisms isolated in culture

\begin{tabular}{lcc}
\hline Organism & Number of cases & Percentage (\%) \\
\hline E Coli & 142 & 86 \\
K Pneumoniae & 11 & 6.7 \\
Acinetobacter & 2 & 1.2 \\
Proteus Vulgaris & 3 & 1.8 \\
Staphylococcus epidermidis & 2 & 1.2 \\
Pseudomonas aeroginosa & 5 & 3 \\
Total & $\mathbf{1 6 5}$ & $\mathbf{1 0 0}$
\end{tabular}

of the world irrespective of the age and sex. We discuss the relationship between age, sex, pathogens isolated, antibiotic sensitivity pattern and associated diseases in UTI. The culture positive cases were 165 (17.4\%) out of 947 cases which were enrolled in our study. Edirisinghe et $\mathrm{al}^{2}$ and Banerjee et al6 in their studies showed culture positive in $31 \%$ and $24.5 \%$ cases respectively. In another study, culture positive as low as $8.7 \%$ has been reported by Mansour et al. ${ }^{9}$

Out of the culture positive samples, female patients were more $(84.8 \%)$ than male patients $(15.2 \%)$. Several other studies show similar findings. ${ }^{6,910}$ The high incidence in female is due to colonization with enteric bacteria due to short urethra. ${ }^{10}$ Most number of positive urine cultures $(33.3 \%)$ was seen in age group 21- 30 years and most of the 
Table 4: Antibiotic sensitivity pattern of organism

\begin{tabular}{lcc}
\hline Name of antibiotic & Number & Percentage (\%) \\
\hline Amoxycillin & 100 & 60.6 \\
Cotrimoxazole & 78 & 47.3 \\
Ofloxacin & 83 & 50 \\
Ciprofloxacin & 83 & 49.7 \\
Cefixime & 80 & 48.5 \\
Nitrofurantoin & 98 & 59.4 \\
Ceftriaxone & 121 & 73.3 \\
Amikacin & 93 & 56.4 \\
\hline Piperacillin- tazobactum & 121 & 73.3 \\
\hline Gentamicin & 93 & 56.4 \\
\hline
\end{tabular}

Table 5: Urine culture and disease association

\begin{tabular}{lcc}
\hline Disease & No of cases & Percentage (\%) \\
\hline UTI (Cystitis, pyelonephritis) & 420 & 44.4 \\
PID & 202 & 21.3 \\
BPH & 92 & 9.7 \\
Nephrolithiasis & 65 & 6.9 \\
\hline PUO & 41 & 4.3 \\
Pregnancy & 127 & 13.4 \\
Pregnancy & 127 & 13.4 \\
Total & $\mathbf{9 4 7}$ & $\mathbf{1 0 0}$
\end{tabular}

patients were females. This showed that UTI is common in reproductive age group which is comparable to studies done by Banerjee et $\mathrm{al}^{6}$, Obiogbolu et $\mathrm{al}^{10}$, Shahina et $\mathrm{al}^{11}$.

The most common organism isolated in our study was $\mathrm{E}$ coli $(86.1 \%)$ followed by K Pneumoniae $(6.7 \%)$ which is in accordance to a study done by Humayun et $\mathrm{al}^{4}$ and Mansour et $\mathrm{al}^{9}$ where $\mathrm{E}$ coli $(70 \%$ and $59 \%$ ) followed by $\mathrm{K}$ Pneumoniae (14\% and $11.6 \%)$ was isolated. There were many other studies which showed that $\mathrm{E}$ coli is the most common causative organism for UTI. ${ }^{2,5-9}$ Pathogens like E coli are associated with population acquired acute uncomplicated infection where as Klebsiella and Enterococcus are known to confer uncomplicated cystitis and pyelonephritis. ${ }^{?}$

Among the antibiotics, Piperacilin- tazobactum (73.3\%) and Ceftriaxone (73.3\%) showed the highest number of sensitivity. Study done by Niranjan et $\mathrm{al}^{12}$ also showed that Piperalcillin - tazobactum $(78.2 \%)$ formed the major antibiotic sensitive to organism isolated. Contrast to our study, Ceftriaxone was resistant (71.4\%) in a study done in India. ${ }^{12}$ In another study done by Nalini et al Ceftriaxone sensitivity was as low as $17.5 \%$ as compared to our study $(73.3 \%)^{13}$

Amoxycillin sensitivity was $60.6 \%$ in our study where as other studies showed resistance to amoxycillin as high as $77.4 \%$ and $84 \% .^{5,14}$ Sensitivity to Amikacin was $56.4 \%$ in our study which is low compared to study done by Niranjan et al where sensitivity was as high as $82.6 \% .^{12}$

Patients with clinical diagnosis of cystitis, pyelonephritis (uncomplicated UTI) formed the major bulk in our study. Similar finding was observed in studies done by Bates et $\mathrm{al}^{15}$. Female with PID and pregnancy formed the second cause of UTI as seen in one study done in Nigeria. ${ }^{10}$ Association of UTI and nephrolithiasis is not very common as seen in our study $(6.9 \%)$ and in study done by Holmgrem et al (7\%). ${ }^{16}$

\section{CONCLUSION}

Though UTI is a common problem in our part of the world, its recognition, proper diagnosis with urine culture and starting appropriate antibiotics according to the culture report plays a major role in preventing an uncomplicated UTI going into a complicated one. E coli is the most common organism isolated in urine culture in most of the hospitals. However the antibiotic sensitivity pattern differs depending on the choice of antibiotic disc. Antibiotic sensitivity pattern in a particular area will give an idea to clinicians regarding empirical treatment of UTI before the availability of laboratory reports.

\section{REFERENCES}

1. Tada GD, Gandhi JP, Patel NK. A study on antibiotic related resistance in UTI patients: a comparison between community acquired and hospital acquired E coli. Nat J Com Med 2012;3:255-8.

2. Edirisinghe LU, Vidanagama D. A retrospective analysis of urine culture results issued by the microbiology department, Teaching Hospital, Karapitiya. Galle Med J. 2008;13: 40-4.

3. Peleg AY, Hooper DC. Hospital acquired infections due to gram negative bacteria. N Engl J Med. 2010;362:1804-13. Crossref

4. Humayun T, Iqbal A. The culture and sensitivity pattern of urinary tract infections in females of reproductive age group. Ann Pak Inst Med Sci. 2012;8:19-22.

5. Shalini, Joshi MC, Rashid MK, Joshi HS. Study of antibiotic sensitivity pattern in urinary tract infection at a tertiary hospital. Nat J Integ Res Med. 2011;2: 43-6.

6. Banarjee S. The study of urinary tract infections and antibiogram of uropathogens in and around Ahmadnagar, Maharashtra. The Internet J Inf Dis. 2009;9:1-5.

7. Vasudevan R.Urinary tract infection: an overview of the infection and the associated risk factors. J Microbiol Exp 2014,1:1-15. Crossref

8. Arslan B, Kozacioglu Z, Ergin OY, Bozkurt IH. Degirmenci T, Yonguc T, Gunlusoy B. Pathogen bacteria of the urinary tract isolated fromm urine cultures and their susceptibility. Erciyes Med J. 2014;36:29-34. Crossref

9. Mansour A, Manizeh M, Zohreh P. Study of bacteria isolated from urinary urinary tract infections and determination of their susceptibility to antibiotics. Jundishapur J Microbiol. 2009:3;118-23.

10. Obiogbolu $\mathrm{CH}$, Okonko $\mathrm{IO}$, Anyamere $\mathrm{CO}$ et al. Incidence of urinary tract infections (UTIs) among pregnant women in Akwa metropolis, Southeastern Nigeria. Sc Res Essay. 2009;4:820-4.

11. Shahina Z, Islam Mj, Abedin J, Chowdhury I, Arifuzzaman M. A study of antibacterial susceptibility and resistance pattern of E coli causing urinary tract infection in Chittagong, Bangladesh. Asian J Biol Sc. 2011;4:548-55. Crossref 
12. Niranjan V, Malini A. Antimicrobial resistance pattern in Escherichia coli causing urinary tract infection among patients. Indian J Med Res. 2014;139:945-8. Crossref

13. Nalini R, Ramya ZE, Meenakshi B, Palniappan N, Poongodi S. Recent sensitivity pattern of Escherichia coli in urinary tract infection. Research and Reviews: J Microbiol Biotec. 2014. Crossref

14. Sohail M, Khurshid M, Saleem HGM, Javed H, Khan AA. Characteristics and antibiotic resistance of urinary tract pathogens isolated from Punjab, Pakistan. Jundishapur J Microbiol. 2015; 8: e19272 Crossref

15. Bates BN. Interpretation of urinalysis and urine culture for UTI treatment. US Pharm. 2013;38:65-8.

16. Holmgren K. Urinary calculi and urinary tract infection. A clinical and microbiological study. Sc J Urol Nephrol Suppl. 1986; 98:1-71. Crossref 